\title{
IMPACT PRESSURE DISTRIBUTION ON A MONOPILE STRUCTURE EXCITED BY IRREGULAR BREAKING WAVE
}

Duje Veic

Wojciech Sulisz

Institute of Hydro-Engineering, Polish Academy of Sciences, Poland

\begin{abstract}
The problem of impact pressure distribution on a monopole structure excited by irregular breaking waves is investigated. The analysis is performed by applying a numerical model that combines potential flow model with a Navier-Stokes/VOF solution. The temporal pressure distribution is analysed for two breaking wave cases characterized by the significant difference in the steepness of the wave front. The peak impact pressures are observed in the region below the overturning wave jet where the pressure increases rapidly resulting in a peak value of the slamming coefficient equal to $C s=2 \pi$. The vertical load distribution provided by the derived model is more realistic than a rectangular shape distribution applied in engineering practice. This is because the vertical load distribution strongly depends on breaking wave shape and it is difficult to uniquely approximate such a load distribution by a rectangle.
\end{abstract}

Keywords: impact pressure distribution, monopile structure, breaking wave slamming process

\section{INTRODUCTION}

The monopile structure is a typical offshore wind tower foundation, which provides the most cost efficient solution for water depths from 15 to $45 \mathrm{~m}$. In these relatively shallow waters, storms can induce large breaking wave impact loading, which needs to be assessed during the design stage. The hydrodynamics of the breaking wave interaction with a monopile foundation is a complex 3-D phenomenon, influenced by the non-linear wave kinematics. This complex phenomenon is usually simplified by $2-\mathrm{D}$ theoretical approaches e.g. Wienke (2001). However, in order to increase the knowledge on breaking wave impact, a study on the 3-D pressure distribution during the wave impact is essential.
Laboratory analyses are very challenging and often provide results with significant scatter, e.g. studies of Zhou et al. (1991) and Chan et al. (1995). Furthermore, the spatial resolution of pressure measurements is too low for complete understanding of the breaking wave impact phenomenon. In fact, the highest resolution of pressure measurement available in the literature, is every $\alpha=10^{\circ}$ around the span of the cylinder (Hildebrandt \& Schlurmann 2011), which is not sufficient to determine vertical impact load characteristics.

In order to obtain insight into the breaking wave slamming process, a numerical model that combines potential flow model with a Navier-Stokes VOF solution is applied. The application of the numerical models enables us to evaluate impact pressures on the structure with high spatial and 
temporal resolution. The numerical models that could be found in literature are mainly focused on the analysis of the impact force considering different stages of the breaking wave impact, e.g. Xiao and Huang (2014), Kamath et al. (2015), and Bihs et al. (2015). However, the pressure distribution during the wave impact is rarely discussed in numerical analysis. In recent study of Ghadirian et al. (2016), discussion on the impact pressure distribution was mainly related to the validation of the numerical model. More detailed discussion on the impact pressure distribution during the wave impact provide Hildebrandt \& Schlurmann (2012). Both aforementioned studies on impact pressure distributions are based on analysis of one breaking wave case. Dependency between the impact pressure distribution and the shape of the breaking wave profile, according to the author's knowledge, has not been discussed so far.

This paper presents numerical analysis on temporal impact pressure distribution on a monopile structure for two breaking wave cases characterized by different shape of wave profiles. The computed impact loads on a monopile structure are presented by applying a vertical slamming coefficient distribution $C_{s}(\mathrm{z})$. Considering a procedure applied in engineering practice, the area under the $C_{s}(\mathrm{z})$ distribution is approximated by a rectangular-shape defined by the slamming coefficient $\left(C_{s s}\right)$ along the impact area $(A)$. This study addresses importance of the breaking wave shape in the determination of $C_{s r}$ and $A$ parameters. Results from this study are compared with an approximate solution suggested by Wienke (2001).

\section{TYPICAL DESIGN PROCEDURE}

This section briefly describes the procedure for calculation of hydrodynamic loads related to the breaking wave impact on monopile structures. Typical industry standard is based on the embedded stream function wave approach, where the highest wave from irregular wave train is selected and modelled by a stream function wave theory. For a small ratio between the diameter of the monopile and the wavelength, the hydrodynamic load is usually estimated according to the wellknown equation presented by Morison et al. (1950). However, in the case of breaking waves, the structure is additionally excited by the impact load, which is out of the scope of Morison's equation. For the estimation of hydrodynamic load excited by the breaking wave on a cylinder, the Morison's equation is extended with additional impact force component

$$
F_{\text {wave_breaking }}=\underbrace{F_{d}+F_{m}}_{\text {Morison }}+\underbrace{F_{i}}_{\text {impact }}
$$

According to the design standard of IEC (2009), the impact force is calculated from the approximate solution proposed by Wienke (2001). For calculation of the impact force $F_{i}(t)$, Wienke (2001) suggested the rectangular distribution of the 2-D line forces $f_{i}(t)$ along the area of the impact, as it is illustrated in Fig. 1a. The impact force $F_{i}(t)$ is approximated by

$$
F_{i}(t)=f_{i}(t) A_{v r t i c a l}=f_{i}(t) \lambda \eta_{b}
$$

where $\lambda$ is the curling factor, which indicates the vertical area of the impact, or how much of the wave crest $\eta_{b}$ is contributing to the impact force, and $f_{i}(t)$ is 2-D line-force (Fig. 1). The formula for the 2-D line-force $f_{i}(t)$ is

$$
f_{i}(t)=C_{s}(t) \rho R c_{b}^{2}
$$

where $C_{s}(t)$ is the slamming coefficient, $R$ is the radius of the cylinder, and $c_{b}$ is the wave phase speed. Theoretical value of the slamming coefficient is presented in Fig. 1b. In this study, the slamming coefficient, which refers to the rectangular line force distribution, is described by $C_{s r}$. The curling factor is usually estimated semi-empirically as $F_{\text {imax,measured/fmax }}$. For the case of the plunging wave breakers, Wienke \& Oumeraci (2005) and Goda et al. (1966) suggested that $\lambda=0.4-0.6$.

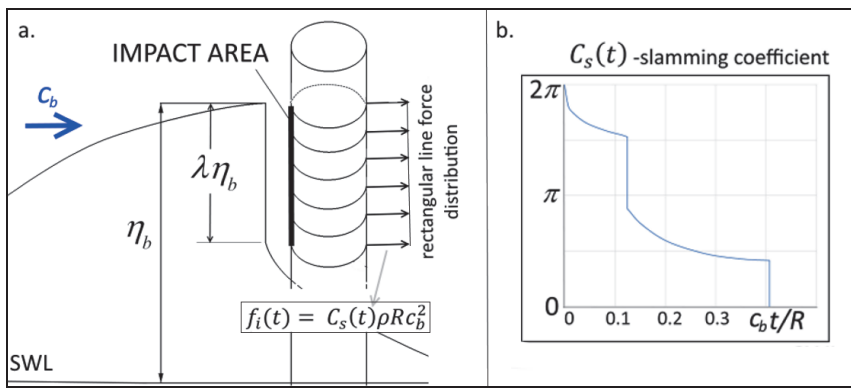

Fig. 1 Schematic view related to Wienke et al. (2001) formulation

\section{THE SELECTED CASE STUDY}

The wave loads are estimated on the bases of the storm occurring once per 50 years. Wave conditions are idealized by assuming JONSWAP energy spectrum. Parameters of the spectrum are $H_{s}=10 \mathrm{~m}, T_{p}=13 \mathrm{~s}$ and $\gamma_{J}=3.3$. The duration of the sea storm is 3-hours.

The simulation of irregular wave propagation is conducted by applying the numerical toolbox OceanWave3D (OCW3D) developed by Engsig-Karup, et al. (2009). OCW3D is fully non-linear potential flow model which solves propagation of regular and irregular waves. OCW3D provides accurate and reliable results Paulsen (2013), Ghadirian et al. (2016 \& 2017). This numerical model, however, is not suitable for solving near-breaking and breaking wave cases. In the case of breaking wave, the numerical stability is ensured by implementing an additional function which artificially reduces the energy of waves whose vertical water particle acceleration exceeds a certain limit (Paulsen 2013)

$$
\frac{d w}{d t}<\gamma g \text { with } \gamma \in[0.4: 1]
$$

In this study, the OCW3D solver is used to identify the highest wave during the 3-hour storm which would break and eventually induce the significant impact loading on a 
structure. Numerical simulations are conducted in a 2-D domain of length $3000 \mathrm{~m}$ and depth $d=30 \mathrm{~m}$. In order to identify the occurrence of a extreme wave which have breaking wave characteristics, the simulations are conducted for two values of the breaking wave filter $\gamma=0.9$ and $\gamma=0.4$ (Fig. 2). This provides time window for the application of the Navier-Stokes VOF model.

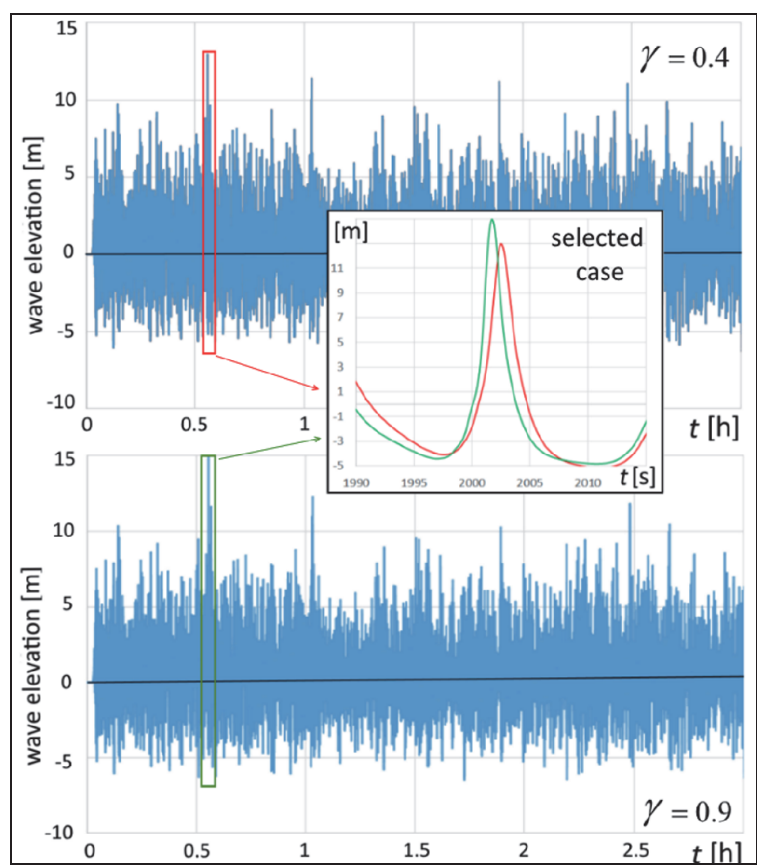

Fig. 2 Irregular wave train simulated by the numerical model

\section{NUMERICAL MODEL}

A numerical model is based on the decomposition strategy proposed by Paulsen et al. (2014), where the outer region is solved within the framework of potential flow solver OceanWave3D and inner region within the framework of the open-source computational fluid dynamics toolbox OpenFoam ${ }^{\circledast}$.

The incompressible Navier-Stokes VOF set of equations are discretized using a finite volume approximation on generally unstructured grids. The solutions in relaxation zones are relaxed towards a solution from the potential flow model (Fig. 3). The slip condition are applied on the whole structure surface. This is because in the wave breaking zone the shear forces on a monopile structure and the pressure forces due to viscous effect are negligible, while below the wave breaking zone inertia forces dominate due to small Keulegan-Carpenter number $(\mathrm{KC}<13)$. Slip boundary conditions are also applied along the seabed. The time step is controlled by adaptive time stepping procedure based on Courant-Friedrichs-Lewy criterion. For all the computations, the maximum Courant number is kept below 0.2. Governing equations are solved within 2 nd order discretisation scheme.

To achieve numerical convergence a very fine computational grid refinement near boundary region of the monopile structure is necessary $(d x \approx 0.5 \% \mathrm{D})$, Veic (2018). The numerical domain used for analyses in this study is constructed with nearly 4.5 million of computational cells (Fig. 3, level 4). The presented numerical model is successfully validated considering the laboratory measurements of total impact force, pressures at the front line of the monopile, and wave elevation, Veic (2018).

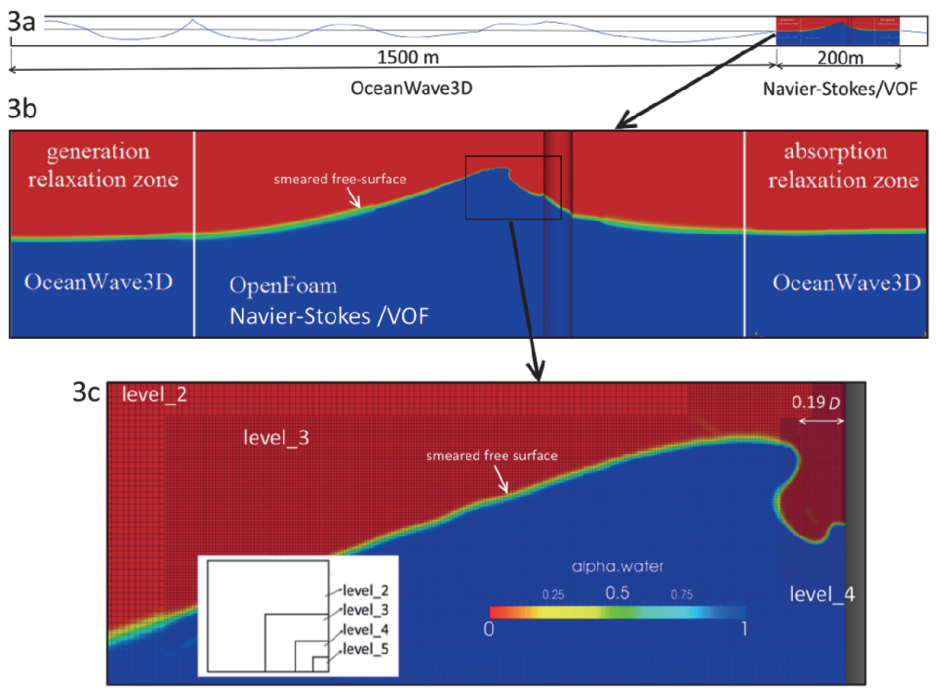

Fig. 3 Sketch of the decomposed numerical domain, water-air interface and unstructured computational grid refinement; level 5 corresponds to $d x=d y=d z=0.25 \% D$

\section{RESULTS}

The mechanism of the wave impact mainly depends on the shape of the wave front and the wave celerity at breaking (Chella, 2016). The wave profile near the location of breaking is asymmetric and characterized by the steep wave front. Breaking waves with the steeper front interacts with the structure in a larger area of the impact and the curling factor is larger.

In the scope of this paper two breaking wave cases are analysed, which are characterized by significant difference in the shape of the wave profile. The case 1 refers to irregular wave breaks over a flat seabed (Fig. 2). The case 2 refers to the identical irregular wave train as in the case 1 , however propagating over the slopped seabed. For this case wave breaks at the water depth of $15 \mathrm{~m}$. Breaking wave profiles are presented in Fig. 4 and corresponding wave parameters are defined in Table 1.

Tab. 1 The asymmetric parameters of the breaking wave profile

\begin{tabular}{|c|c|c|c|c|c|c|}
\hline & $\mathrm{H}$ & $\eta \mathrm{b}$ & $\varepsilon$ & $\delta$ & bed-type & depth \\
\hline & {$[\mathrm{m}]$} & {$[\mathrm{m}]$} & {$[/]$} & {$[/]$} & {$[/]$} & {$[\mathrm{m}]$} \\
\hline 1 & 15.9 & 12.8 & 0.55 & 0.28 & flat & 30 \\
\hline 2 & 15.7 & 9.9 & 2.43 & 0.18 & slope $(1: 10)$ & 15 \\
\hline
\end{tabular}




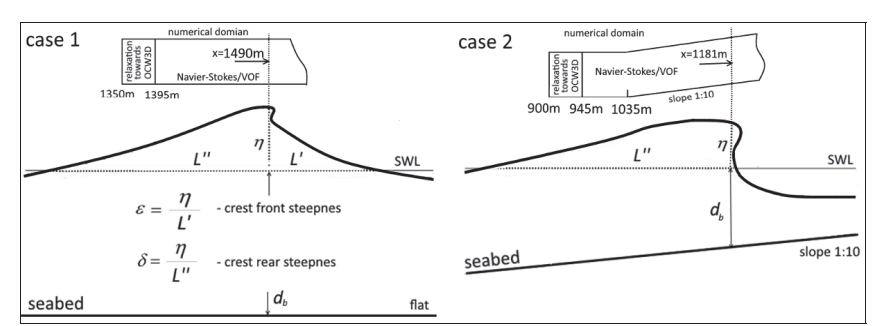

Fig. 4. The breaking wave shapes with corresponding wave asymmetric parameters

In the studies of Wienke et al. (2001) and Chan et al. (1995), the maximum impact force is identified for the stage when wave breaks slightly before the structure. In such scenario, the breaker tongue hits the cylinder just below the wave crest level. This stage of the breaking wave impact is analysed in this study. The length of the overturning wave jet before impact on the structure is $l_{1}=0.13 R$ and $l_{2}=0.26 R$ for the case 1 and the case 2, respectively (Fig. 5).

Velocity distribution in the overturning wave crest for the aforementioned braking wave stage is shown in Fig. 5. When the water particle velocity exceeds the wave celerity, the wave crest propagates forward in the form of an overturning jet. In this study, the breaking wave celerity is identified as the horizontal particle velocity at the toe of the overturning jet (Fig. 5). Approaching the shallow water, the wave height and the particle velocity at the wave crest increase while the wave length and wave celerity decrease. Therefore, the breaking wave celerity for the case 1 is higher than for the case 2 . The breaking wave celerity for the case 1 is $c_{b 1} \approx 17.4 \mathrm{~m} / \mathrm{s}$ while for the case 2 is $c_{b 2} \approx 16.77 \mathrm{~m} / \mathrm{s}$.

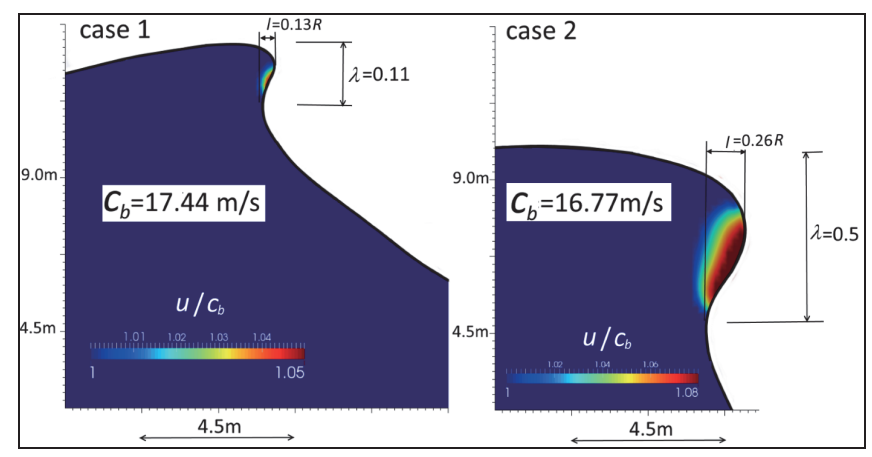

Fig. 5 Wave kinematics for the overturning wave crest, approximation of the overturning wave crest length $(l)$ and the vertical impact area $(\lambda)$

The hydrodynamic forces are computed for the monopile with the uniform diameter of $7.2 \mathrm{~m}$ (Fig. 6). The results show that the magnitude of impact force is higher than the magnitude of non-impact force in both analysed cases. In the case 1 wave kinematics is well approximated by the stream function wave theory, so the Morison's equation provides good approximation for the non-impact part of the force. In the case 2 wave elevation is very asymmetric and beyond the scope of the stream function wave theory. The results show that the crest height in the case 1 is $25 \%$ higher than in the case 2 , while the impact area on the structure is much higher in the second case. The curling factor is estimated from the toe of the overturning jet to the wave crest height (Fig. 5). The curling factor for the case 1 is $\lambda_{1}=0.14$ while for the case 2 is $\lambda_{2}=0.5$. The impact force is identified from the moment when dynamic pressure on the structure in the impact area exceeds $\mathrm{p}=0.5 \rho c_{b}^{2}$. The analysis shows that the magnitude of the computed impact force for the case 2 is $30 \%$ higher than for the case 1 (Fig. 6).

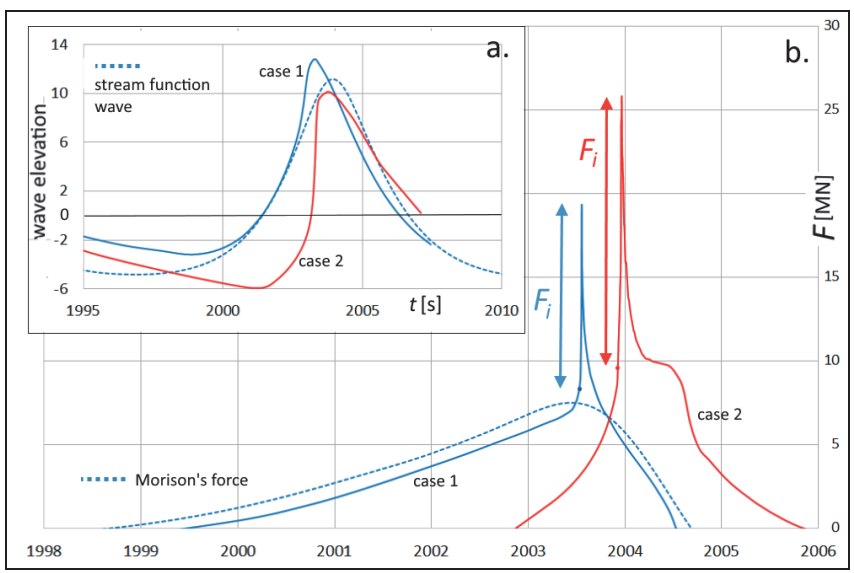

Fig. 6 Wave elevation (a) and hydrodynamic load on the monopile structure (b)

The maximum impact force is normalized by assuming the rectangular impact load distribution along the impact area (Fig. 1), $C_{s r}=F_{\text {imax }} / \rho \eta_{b} \lambda R c_{b}{ }^{2}$. The corresponding slamming coefficient for the case 1 is $C_{s r 1}=1.8 \pi$ while for the case 2 is $C_{s r 2}=\pi$. The theoretical slamming coefficient proposed by Wienke (2001) is $C_{s r}=2 \pi$. Figure Fig. 7 shows comparison between computed impact forces and the results of an approximate solution of Wienke (2001). In addition to the differences in the magnitude of impact force, the temporal characteristic of the impact force are also different in both approaches. The rise phase of the impact force, defined by the rise time $\left(t_{F i}\right)$, is out of the scope of Wienke solution. In the case 1 the peak of computed impact force is comparable to the Wienke approximation, however, the decay of the computed impact signal part is several times faster than in Wienke approximation. The effects of presented differences on the structure response is a matter for discussion for structural analysis, which is out of the scope of this paper.

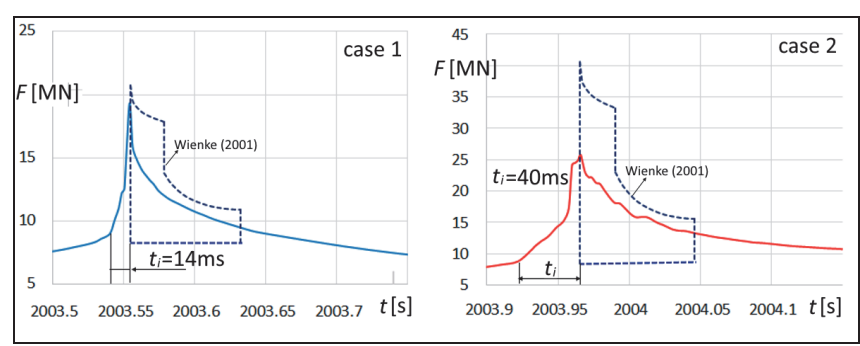

Fig. 7 Comparison between the computed impact force and analytically calculated according Wienke (2001) 
In order to better understand the mechanism of the wave impact on the structure, a temporal impact pressure distribution on the structure is investigated. The impact pressure distribution for different moments of wave impact is presented in snapshots of Fig. 8. The pressure is normalized by $\rho c_{b}^{2}$. The presented snapshots $t_{a 1}$ and $t_{a 2}$ refers to the initial stage of wave impact. The initial stage of impact for the case 1 is the result of the interaction between the breaking wave tongue and the wave run up on the structure. The observed peak pressure is in the range of $2 \rho c_{b}^{2}$. The initial stage of the impact for the case 2 is the result of the interaction between the breaking wave tongue and the structure. The observed peak pressure is in the range of $5 \rho c_{b}^{2}$.

The presented snapshots $t_{b 1}$ and $t_{b 2}$ corresponds to the time between the initial stage of the impact and the time of the peak impact force. The snapshot $t_{b 1}$ shows beginning of the
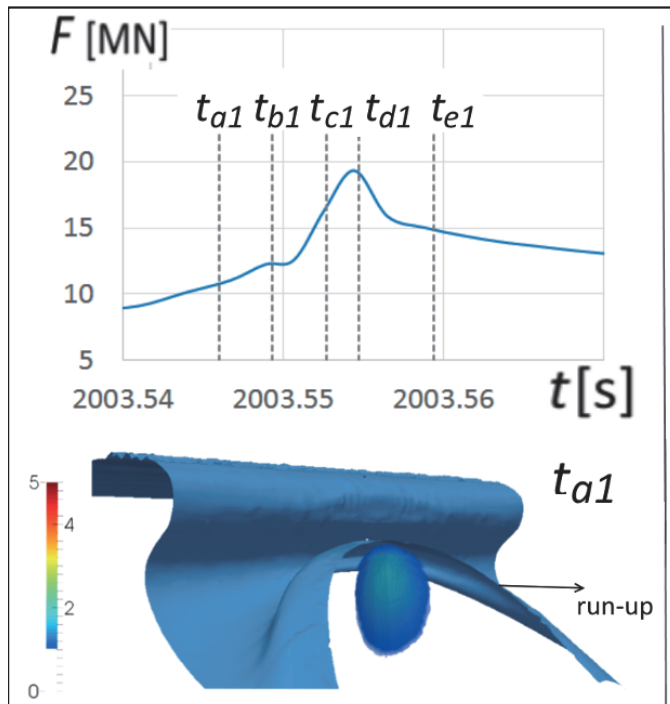

0

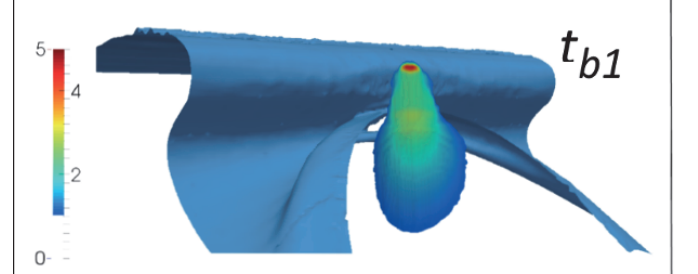

0
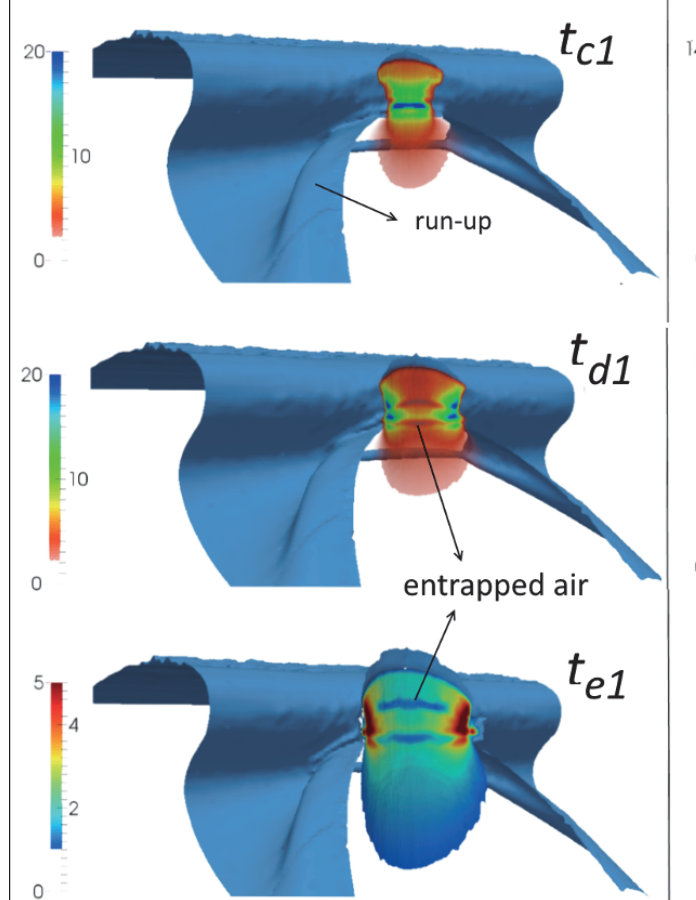
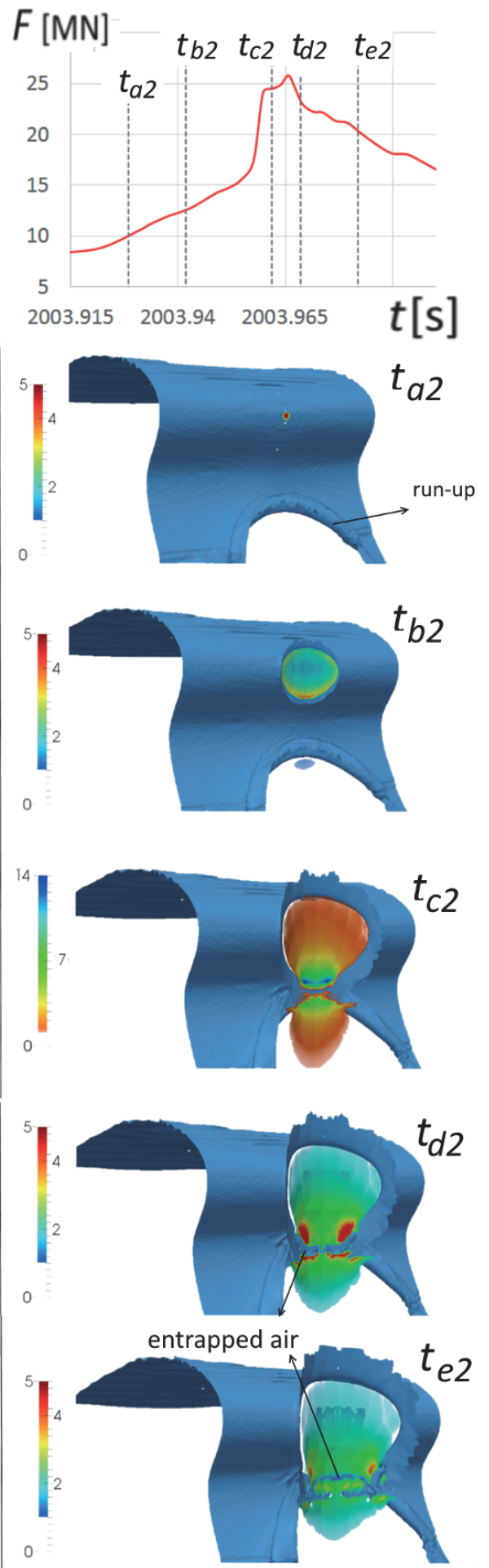

Fig. 8 Temporal dynamic pressure distribution during the selected moments of the wave impact interaction between the breaking wave tongue and the structure. The observed peak pressure is in the range of $5 \rho c_{b}^{2}$, the same as for the snapshot $t_{a 2}$. The snapshot $t_{b 2}$ shows the interaction of the breaking wave tongue with the structure at the higher azimuth angles. Because of the higher angle of attack between the propagating overturning wave crest and the structure, impact pressures around the span of the structure are lower, and are in the range of $3 \rho c_{b}^{2}$. At the equivalent moment, the impact pressures at the front line of the structure are in a decay phase, and are in the range of $\rho c_{b}^{2}$.

The presented plots $t_{c 1}$ and $t_{c 2}$, as well as the plots $t_{d 1}$ and $t_{d 2}$, corresponds to the time around the peak of the impact force. Higher impact pressures are observed for $t_{c}$, while for $t_{d}$ a higher impact area is observed which results in similar magnitude of the impact force. The maximum impact pressures on the structure are observed in the region below the overturning wave jet, where overturning wave jet meets the wave run-up. At the selected moments $t_{c 1}$ and $t_{c 2}$, the peak impact pressures are in the range of $20 \rho c_{b}^{2}$ and $14 \rho c_{b}^{2}$, respectively, which are of the same order of magnitude as observed in laboratory study of Zhou et al. (1991) and Chan et al. (1995). When the breaking wave tongue hits the structure, the impact energy dissipates. Majority of the impact energy is transformed into the kinetic energy of water jets, which are generated in all directions. The speed of water jets is several time faster than the speed of the wave propagation. When high speed 
water jets interacts with the wave run-up on the structure the impact energy dissipation is significantly reduced due to the fact that generation of water-jets is limited. This is the region of the highest impact pressure.

The distribution of the load is determined by integrating the pressure around the strips of the monopile structure. The vertical distribution of the load and the corresponding slamming coefficient can be obtained by dividing the monopile structure into small strips $(d z=90 \mathrm{~mm})$, integrating pressure over the each strip, subtracting the non-impact part of the force, and normalizing calculated strip forces by $\rho R c_{b}^{2} d z$. The vertical distribution of the slamming coefficient is presented in Fig. 9. The peak slamming coefficients are detected in the zone of the highest pressure area, as it was presented in Fig. 8. The peak slamming coefficient is $C_{s}=2 \pi$ for both analysed cases. Away from the peak region, slamming coefficient decays rapidly. The area of the impact load on the structure is significantly higher than the impact area which is usually defined by the curling factor.

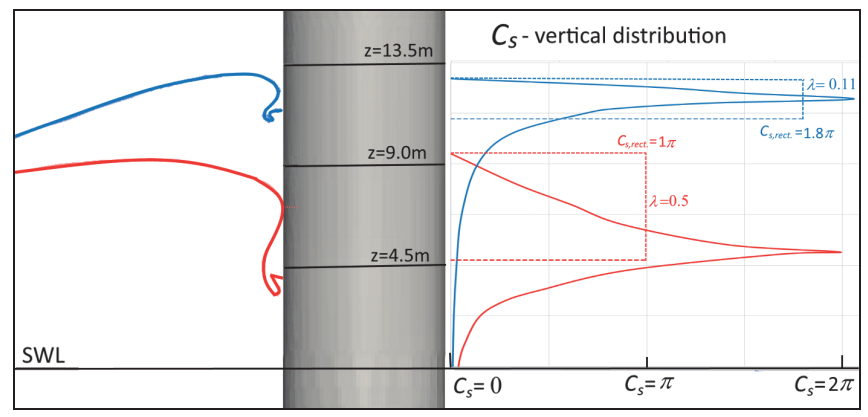

Fig. 9 The slamming coefficient and corresponding area of rectangular distribution defined by $C_{s r}$ and $\lambda$

The engineering practice approximates the area under the vertical slamming coefficient by a rectangle (Fig. 9). The problem is that $C_{s r}$ and $\lambda$ values are not uniquely defined in the literature. This is illustrated in Fig. 9 which shows that by assuming geometrically defined parameter $\lambda$ (Fig. 5), $C_{s r}$ is a function of the wave shape. This leads to non unique and confusing results.

Additional analyses indicate that $C_{s}$-vertical distribution for breaking waves characterized by the parameter of the crest front steepness $(\varepsilon)$ in the range of 0.55 (case 1) to 2.43 (case 2), fits into the boundaries between two curves presented in Fig. 10. Therefore, the focus of the future work is to identify the unique $C_{s}(\mathrm{t})$-vertical characteristics, which would present the best approximation for analysed $\varepsilon$-range. The derived model enable us to determine such a distribution for engineering applications which confirm preliminary results. However, more studies are required to derive more precise results.

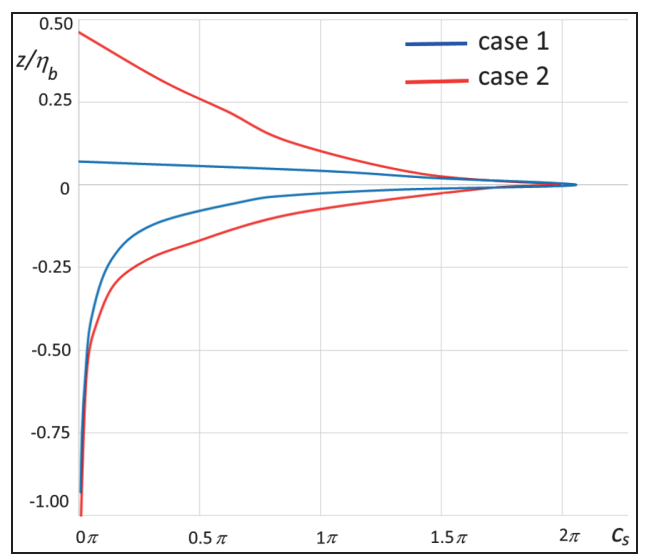

Fig. 10 The vertical slamming coefficient for the normalized vertical position (zero ordinate corresponds to the vertical location of the highest impact pressures)

\section{CONCLUSIONS}

The problem of the impact of irregular breaking waves on a monopole structure is investigated. The numerical model, which provides high spatial and temporal pressure distribution on the monopile structure arising from wave impact, is derived. The temporal pressure distribution is analysed for two breaking wave cases characterized by significant differences in the steepness of the wave front. The analysis focuses on the stage of wave impact for which the overturning wave jet hits the structure just below the wave crest level.

The results show a similar mechanism of the breaking wave impact on a structure in both impact cases. The peak impact pressures is detected in the region below the overturning jet, where the overturning wave jet meets the wave run-up on a structure. The impact pressures in this region are more than 4 times higher in comparisons with the pressures at the beginning of the wave impact when the tongue of the overturning jet hits the structure.

The analysis shows that the value of the peak slamming coefficient is approximately equal to $C_{s}=2 \pi$. Away from the peak region, slamming coefficient decays rapidly. The area of the impact load on the structure is significantly higher than the impact area defined by the curling factor.

A rectangular distribution of loads applied in engineering practice to approximate the maximum impact force results in the non-unique outcome. This is because the parameters, which defined the rectangular distribution, depend on breaking wave shape which changes from case to case and it is difficult to define them unequally beforehand.

\section{ACKNOWLEDGMENT}

A part of this work was carried out in the framework of MareWint project under research area FP7-PEOPLE-2012ITN Marie-Curie Action: "+Initial Training Networks" conducted in CTO, Gdansk, Poland. 


\section{REFERENCES}

1. Bihs, H., Kamath, A., Alagan Chella, M., \& Arntsen, Ø. A. (2016). Breaking-Wave Interaction with Tandem Cylinders under Different Impact Scenarios. Journal of Waterway, Port, Coastal, and Ocean Engineering, 04016005.

2. Chan, E. S., Cheong, H. F., \& Tan, B. C. (1995). Laboratory study of plunging wave impacts on vertical cylinders. Coastal Engineering, 25(1), 87-107.

3. Chella M.A. (2016). Breaking Wave Characteristic and Breaking Wave Forces on Slender Cylinders, Ph.D. at NTNU

4. Engsig-Karup, A. P., Bingham, H. B., \& Lindberg, O. (2009). An efficient flexible-order model for 3D nonlinear water waves. Journal of computational physics, 228(6), 2100-2118.

5. Ghadirian, A., Bredmose, H., \& Dixen, M. (2016, September). Breaking phase focused wave group loads on offshore wind turbine monopiles. In Journal of Physics: Conference Series (Vol. 753, No. 9, p. 092004). IOP Publishing.

6. Goda, Y., S. Haranaka, and M. Kitahata (1966). Study of impulsive breaking wave forces on piles. Report Port and Harbour Technical Research Institute 6.5, 1-30

7. Hildebrandt, A., \& Schlurmann, T. (2012). Breaking Wave Kinematics, local Pressures and Forces on a Tripod Support Structure. In Proceedings of the Coastal Engineering Conference, No. 33

8. IEC (2009), International Electrotechnical Commission, IEC 61400-3, Wind Turbines, Part 3.

9. Kamath, A., Chella, M. A., Bihs, H., \& Arntsen, Ø. A. (2015). Breaking Wave Interaction with a Vertical Cylinder and the Effect of Breaker Location. CFD based Investigation of Wave-Structure Interaction and Hydrodynamics of an Oscillating Water Column Device, 171.

10. Morison, J. R., Johnson, J. W., \& Schaaf, S. A. (1950). The force exerted by surface waves on piles. Journal of Petroleum Technology, 2(05), 149-154.

11. Paulsen (2013), Efficient computations of wave loads on offshore structures, PhD thesis, DTU -Department of Mechanical Engineering

12. Paulsen, B. T., Bredmose, H., \& Bingham, H. B. (2014). An efficient domain decomposition strategy for wave loads on surface piercing circular cylinders. Coastal Engineering, 86, 57-76.

13. Veic D (2018), Effect of the Breaking Wave shape on the Temporal and Spatial Pressure Distribution around a
Monopile Support Structure, Ph.D.

14. Wienke, J. (2001). Druckschlagbelastung auf schlanke zylindrische Bauwerke durch brechende Wellen. Technical University of Braunschweig, Germany.10

15. Wienke, J., \& Oumeraci, H. (2005). Breaking wave impact force on a vertical and inclined slender piletheoretical and large-scale model investigations. Coastal Engineering, 52(5), 435-462.

16. Xiao, H., \& Huang, W. (2014). Three-dimensional numerical modeling of solitary wave breaking and force on a cylinder pile in a coastal surf zone. Journal of Engineering Mechanics, 141(8), A4014001.

17. Zhou, D., Chan, E. S., \& Melville, W. K. (1991). Wave impact pressures on vertical cylinders. Applied Ocean Research, 13(5), 220-234.

\section{CONTACT WITH THE AUTHOR}

\author{
Duje Veic \\ e-mail:dujeveic@ibwpan.gda.pl \\ Wojciech Sulisz \\ e-mail:sulisz@ibwpan.gda.pl \\ Institute of Hydro-Engineering \\ Polish Academy of Sciences \\ 7 Kościerska St. \\ 80 - 328 Gdańsk \\ Poland
}

